\title{
Niveles socioeconómicos-culturales y ejecución lingüística
}

\section{A. Barca Lozano}

R. Santorum Paz

R. González Cabanach

A. Porto Riobó

J. C. Núñez Pérez

Universidad de Santiago de Compostela

\section{INTRODUCCION}

El punto de partida de este estudio se refiere a la asunción de una diversidad lingüística dentro de una comunidad global, condicionada por variables o factores extralingüísticos. Es decir, al hecho de que el lenguaje no desempeña el mismo papel en todas las culturas, ni incluso dentro de una misma cultura entre las capas pertenecientes a diferentes medios sociales.

Otorgamos al lenguaje un papel fundamental en el proceso de aprendizaje escolar, por consiguiente será condición necesaria, aunque no suficiente, para lograr éxito en el sistema educativo.

La conducta lingüística está integrada en una realidad social, en general, $y$ en un contexto sociocultural, en particular, por ello no podemos deslindar su estudio del marco en que se produce y desarrolla. La importancia del medio sociolingüístico y cultural en que se desenvuelve el niño adquiere así gran relevancia al evaluar el lenguaje, dado que el entorno sociocultural puede favorecer o enlentecer los mecanismos, a partir de los cuales se hace efectiva la utilización de esta conducta.

La conducta lingüística, según J. Mayor (1984), se encuentra enmarcada en una serie de coordenadas, la de sus condicionamientos biológicos y socioculturales (H. Giles, W. P. Robinson y P. H. M. Smith, 1980) y la de sus funciones: comunicación y pensamiento.

Bajo la consideración de este esquema, lo que nos interesa en el presente estudio es el análisis de los condicionamientos socioculturales del lenguaje, puesto que nuestra finalidad es comprobar si existen diferencias lingüísticas según la clase social de pertenencia de los sujetos tal y como señalaron $B$. Bernstein (1960, 1972), D. Lawton, 1963), F. Williams y R. Naremore, 1969), G. Turner y R. Pickwance (1971), G. Turner (1972), D. Aram, 1975), entre otros, y en caso afirmativo, analizar qué factores o variables pueden responder de este fenómeno.

La importancia de estudiar el lenguaje en el contexto social en que se produce y desarrolla ha sido señalada por diferentes paradigmas de la psicología, tales como la Teoría Ecolingüística (Salzinger, K., 1979), el paradigma cognitivo (Leontiev, N., 1971; Bronckart, J. P., 1977; Wertsch, J. V., 1978) y el paradigma de la actividad lingüística (Halliday, M. A. K., 1978). Estos paradigmas, al considerar las implicaciones sociales de la conducta lingüística, po- 
nen en evidencia el supuesto clásico, que va de F. de Saussure a N. Chomsky, de la existencia de una comunidad lingüística homogénea y la asunción de variaciones libres y teóricamente insignificantes. Se produce, por tanto, en la Psicología del Lenguaje actual, un desplazamiento hacia las múltiples y recíprocas relaciones entre el lenguaje y la sociedad, específicamente hacia el estudio del contexto sociocultural del lenguaje.

Por su parte las principales teorías de la adquisición y desarrollo del lenguaje, dependiendo del tipo de aproximación que realizan, otorgan al medio -entorno social - diversos niveles de importancia en la configuración y desarrollo de la conducta lingüística.

Así, para los conductistas (B. F. Skinner) el medio será fuente y modelo de una conducta de comunicación, es decir, la contribución del medio es considerada determinante en tanto que agente de selección y refuerzo de conductas verbales y fuente de asociación de un signo a un objeto. Explican la conducta verbal determinando las variables que la controlan, haciendo referencia a la historia de refuerzos del sujeto en el marco del grupo social en el que está inmerso.

La escuela del relativismo lingüístico y cultural (E. Sapir y B. F. Whorf) enfatiza el papel de la cultura en la adquisición del lenguaje. De forma general, la conducta humana está determinada por patrones sociales y culturales, y cada individuo es considerado como el producto de su historia sociocultural específica. El lenguaje es indisociable de fenómeno social ya que se convierte en un instrumento de intercambio social integrado por unidades y símbolos que corresponden a categorías o conceptos establecidos por convención.

Dentro de esta escuela, B. Bernstein, autor especialmente relevante y de referencia obligada en aquellos estudios que, como éste, tratan de realizar una aproximación al lenguaje dentro de los distintos grupos sociales, señala que el código verbal determina el desarrollo cognitivo del individuo y la estructura social contribuye a perpetuarlo. El código lingüístico que adquiere el niño depende del entorno social donde éste se desarrolle, es decir, son las características de los medios sociales las que determinan el uso que del lenguaje hace el sujeto. El lenguaje de un individuo refleja la manera por la que se estructuran sus percepciones, sus acciones y el tipo de interacciones mantenidas por este individuo con los sujetos y objetos que componen su medio. En reusmen, según B. Bernstein, la forma de las relaciones sociales - contexto educativo familiar - que se producen en las estructuras sociales - clases sociales- determinan el modo de utilización del lenguaje - Lenguaje Público/Lenguaje Formal-y éste, la orientación cognitiva del sujeto que, en función de los medios sociales de pertenencia, puede repercutir en un desarrollo simbólico y, por consiguiente, en el éxito escolar o, por el contrario, en un cambio simbólico que se traduce, por lo general, en fracaso en la educación formal.

Para los innatistas (N. Chomsky y D. McNeill) el medio es la condición para el ejercicio de una función cognitiva biológicamente determinada. La importancia del medio es fundamental en la adquisición del lenguaje, pero está desplazada. En ningún caso, el medio lingüístico podrá ser considerado como formando el lenguaje del niño, sin embargo es la condición para el ejercicio de la función lingüística, porque privado de estimulación durante el «periodo crítico», la función desaparece, puesto que es necesario que sea puesta en funcionamiento en el momento adecuado.

Por último, los interaccionistas consideran que el papel del medio es fundamental en la adquisición y desarrollo del lenguaje. El estudio del lenguaje no puede ser analizado de manera aislada, puesto que todos los aspectos del desarrollo están interrelacionados a lo largo de la génesis del individuo. Autores como J. Piaget, A. R. Luria, L. S. Vigotsky, J. S. Brunner y H. Wallon, 
entre otros, resaltan el problema de la interacción entre la representación simbólica, el lenguaje y el medio social. Postulan que el medio juega un papel integrador como consecuencia de que es a la vez fuente, condición y objetivo de la principal actividad de relación.

Todo lo apuntado hasta aquí nos permite señalar que el desarrollo del lenguaje sólo es efectivo por la interacción con el medio debido a que el niño no crece aislado y la lengua que aprende es indisociable de su cultura y del medio físico-social. Los usos que el sujeto hace del lenguaje están en función del lugar geográfico en que vive, del grupo social al que pertenece su familia $y$, dentro del marco familiar, de sus aspiraciones culturales, condiciones económicas, actividad profesional, etc.

No podemos, por tanto, analizar el lenguaje sin tener en cuenta estos factores sociológicos, ya que éstos determinan o condicionan la utilización de unos registros de carácter formal y estructural o de otros más informales, de sintaxis simple y de escasa precisión en los enunciados. Todos los hablantes conocen y utilizan este último tipo de lenguaje, pero para acceder a modos más formalizados de hablar se requiere de la influencia de ambientes con riqueza de intercambios verbales que ofrezcan modelos más ricos y más concretos por lo que respecta al uso de significaciones y al empleo de reglas sintácticas, que no reduzcan las necesidades de comunicación a los aspectos utilitarios de la relación verbal.

Partiendo de esta concepción del estudio del lenguaje, a la cual nos adherimos, desarrollamos la fase empírica de esta investigación, cuyo objerivo prioritario es conocer si las variables/factores socioeconómicos y socioculturales definitorios de las distintas clases sociales predicen diferencias lingüísticas entre los sujetos y determinar cuáles de estas variables están afectando e incidiendo en el nivel de ejecución lingüístico alcanzado por los niños/as pertenecientes a medios sociales diferenciales en una muestra de escolares del $\mathrm{Ci}$ clo Medio de la E.G.B. en Galicia.

\section{METODO}

\section{A. Variables analizadas}

Las variables independientes analizadas, a partir de las cuales predeciremos los resultados alcanzados, hacen referencia a la clase social conceptualizada a través de factores socioeconómicos y socioculturales.

En términos generales, definimos a la clase social como un agregado de individuos o familias que participan de igual o similar prestigio, en función de unos criterios objetivos de valorización dentro de un sistema de estratificación social y en base a su posición dentro del sistema de la división social del trabajo. Este conjunto de individuos disfrutará de un estatus social análogo, una situación social semejante y posiciones sociales parecidas. Específicamente, una clase social se puede describir por el lugar o posición que ocupa un estrato de personas en una escala de prestigio y ocupacional.

Para operacionalizar la clase social construimos un Cuestionario basándonos en indicadores objetivos que recopilamos de la literatura existente sobre este tema.

Las variables seleccionadas y que utilizamos en nuestro Cuestionario son, entre otras, las siguientes: profesión y estudios de los padres, ingresos mensuales familiares, posesión de bienes y equipamiento de la vivienda.

Por lo que respecta a las variables socioculturales consideramos índices tales como: lectura de libros y periódicos, frecuencia en la asistencia al cine, conferencias, actividades culturales, etc. 
Como estas variables son numerosas estudiamos la estructura de las correlaciones que existen entre ellas, de modo que reduzcamos mediante análisis factoriales el número de variables que van a ser utilizadas en el estudio del lenguaje.

Los indicadores de profesión y estudios de ambos padres nos permitieron dividir a los sujetos en tres grupos sociales y realizar los análisis descriptivos. La elección de estos índices como variables criterio está basada en el hecho de que, por un lado, la profesión es un indicador objetivo decisivo que da lugar a unos ingresos, ambos son correlativos y condicionan la pertenencia a una clase social dada; por otro lado, la educación está circularmente relacionada con las clases sociales, puesto que existe un paralelismo entre el nivel social y el educativo, que denota una determinada posición económica.

La variable dependiente que tratamos de explicar fue el lenguaje. Para definirlo de modo operacional utilizamos el Test de Habilidades Psicolingüísticas de Illinois (I.T.P.A.). Esta prueba se refiere a aquellas funciones del lenguaje que permiten captar, interpretar y transmitir información. Implica las habilidades psicolingüísticas necesarias para comprender mensajes -comprensión visual y auditiva-, relacionar conceptos - asociación visual y auditiva-, expresar ideas -expresión verbal y motora-, utilizar la gramática de modo automático e identificar un objeto común a partir de una representación visual incompleta -integración gramatical y visual- y recordar material no significativo -memoria secuencial auditiva y visomotora-.

\section{B. Hipótesis}

En base al planteamiento teórico desarrollado formulamos las siguientes hipótesis:

H.1. Si consideramos que las características del entorno social influyen en la adquisición y desarrollo del lenguaje, entonces cabría esperar que los sujetos de las diferentes clases sociales obtengan distintos niveles en rendimiento en lenguaje.

H.1a. Afirmamos que existen diferencias significativas entre los niños que pertenecen a la clase social baja respecto de los de la clase social media en rendimiento en lenguaje según el I.T.P.A.

H.1b. Afirmamos que existen diferencias significativas entre los niños que pertenecen a la clase social baja respecto de los de la clase social alta en lenguaje.

H.1c. Afirmamos que existen diferencias significativas entre los niños que pertenecen a la clase social media y los de la clase alta, en rendimiento en lenguaje.

Estas subhipótesis pueden resumirse del siguiente modo: las diferencias en cuanto a la ejecución lingüística son más acusadas en la medida en que el nivel socioeconómico y sociocultural de los sujetos de la muestra presenta mayor variablidad.

H.2. En el análisis del lenguaje hay unos indicadores que inciden diferencialmente en el rendimiento del niño en el lenguaje. Afirmamos que las variables definitorias del nivel sociocultural afectan cuantitativamente y en mayor medida, que las variables que definen el nivel socioeconómico, la ejecución linguística de los sujetos de la muestra.

\section{Muestra}

Siguiendo un procedimiento de muestreo estratificado aleatorio, la muestra quedó compuesta por 77 niños y niñas del Ciclo Medio que cursaban 3.9 
y 4. ${ }^{\circ}$ de E.G.B. La elección de los sujetos de este Ciclo estuvo motivada por el hecho comprobado en algunas investigaciones de que en el campo de los aprendizajes de la primera infancia las diferencias de clase social no son muy estables. Por otra parte, la no selección de niños/as de $5 .{ }^{\circ}$ Curso, que también se incluyen en el Ciclo Medio, obedece al hecho de que el instrumento utilizado para evaluar el lenguaje no resulta fiable a partir de los 9.6 años.

TABLA I

Distribución de sujetos por cursos

\begin{tabular}{ccc}
\hline Curso & N & $\%$ \\
\hline 3.9 E. G. B. & 39 & 50 \\
3.9 E. G. B. & 38 & 49 \\
\hline
\end{tabular}

TABLA II

Distribución de sujetos por sexos

\begin{tabular}{lll}
\hline Sexo & N & $\%$ \\
\hline Varón & 42 & 54 \\
Mujer & 35 & 45 \\
\hline
\end{tabular}

La distribución de los sujetos de la muestra por cursos, edades y sexos queda reflejada en las tablas I, II y III.

TABLA III

Distribucion de sujetos por edades.

\begin{tabular}{lrl}
\hline \multicolumn{1}{c}{ Edad } & N & $\%$ \\
\hline 8 & 8 & 10 \\
8.5 & 21 & 27 \\
9 & 30 & 38 \\
9.5 & 18 & 23 \\
\hline
\end{tabular}

Los padres de los alumnos seleccionados también participaron aportando datos a nuestra investigación. A ellos se les envió el Cuestionario de Clase Social para su cumplimentación a fin de establecer diferentes grupos sociales.

De acuerdo con los criterios de profesión y estudios de los padres, se han formado tres grupos de sujetos:

Grupo 1: Integrado por aquellos sujetos que pertenecen a familias en las que los padres desempeñan profesiones sin cualificar o semicualificadas y que no tienen ningún tipo de estudios o sólo los primarios.

Este grupo quedó formado por 34 sujetos y representa 45 por 100 de la muestra. La denominamos Grupo de Clase Social Baja.

Grupo 2: Integrado por aquellos sujetos cuyos padres son cuadros medios o desempeñan profesionales cualificadas y tienen estudios medios o bachillerato.

Este grupo lo integran 18 sujetos y representa el 23 por 100 de total de la muestra. Le denominamos Grupo de Clase Social Media.

Grupo 3: Integrados por aquellos sujetos cuyos padres son cuadros superiores y tienen estudios universitarios.

Este grupo compuesto por 25 sujetos representa el 34 por 100 de la muestra. Le denominamos Grupo de Clase Social Alta.

Los colegios fueron seleccionados aleatoriamente con el propósito de que representaran los distintos estratos sociales. En un principio, con el objeto de obtener una muestra lo más homogénea posible, se eligieron únicamente 
centros públicos, pero dada la dificultad de obtener en ellos sujetos que representaran a la clase social alta, recurrimos a colegios privados. A este respecto quisiéramos señalar que aunque los diferentes centros donde estudian los niños pueden ejercer distintas influencias, éstas, lejos de ser ajenas al entorno socioeconómico, son precisamente parte del mismo (Soriano Llorent, A., 1972).

\section{Instrumentos de medida}

Los instrumentos de medida utilizados fueron, por una parte, el Test de Habilidades Psicolinguísticas de S. Kirk, D. McCarthy y W. Kirk adaptado por TEA Ediciones bajo la dirección de S. Ballesteros y A. Cordero y, por otra parte, el Cuestionario de Clase Social de elaboración propia.

Para la consecución de los objetivos de este trabajo y a fin de poder comprobar o rechazar las hipótesis planteadas utilizamos las técnicas de Análisis Factorial, la Prueba «t» de Student y el Análisis de Regresión Múltiple.

\section{RESULTADOS}

\section{A. Análisis Factorial}

Nuestro propósito al utilizar esta técnica de análisis era comprobar si las variables que suponíamos evaluaban el nivel socioeconómico y el sociocultural se referían todas ellas a estos dos niveles que pretendíamos analizar y delimitar. Además, queríamos conocer en qué grado cada variable predecía o explicaba estos niveles socioeconómico y sociocultural.

El Análisis Factorial se efectuó sobre las respuestas que dieron los padres de 77 niños/as del Ciclo Medio de la E.G.B. que componen la muestra, al Cuestionario de Clase Social; específicamente sobre un total de 35 variables que obtuvimos una vez codificadas las respuestas de los distintos ítems.

Las 35 variables fueron intercorrelacionadas y se analizaron los factores de correlación mediante el método de Componentes Principales. Por último, cuatro factores fueron rotados en forma ortogonal por medio del método $\mathrm{Va}$ rimax.

La naturaleza de las disposiciones factoriales concordó con los resultados esperados puesto que obtuvimos un primer factor que denominamos socioeconómico integrado por 11 variables, con una varianza explicada de 37.23 por 100. Las variables y sus cargas factoriales pueden verse en la tabla IV.

\section{TABLA IV}

Factor 1: Nivel socioeconómico

\begin{tabular}{lc}
\hline \multicolumn{1}{c}{ Variables } & $\begin{array}{c}\text { C. } \\
\text { factoriales }\end{array}$ \\
\hline Profesión del padre & .909 \\
Ingresos familiares mensuales & .901 \\
Estudios del padre & .884 \\
Presupuesto educativo & .868 \\
Estudios de la madre & .832 \\
Equipamiento de la vivienda & .784 \\
Automóvil & .750 \\
Profesión de la madre & .725 \\
Actividades culturales del padre & .665 \\
Actividades culturales de la madre & .653 \\
Número de habitaciones de la vivienda & .646 \\
\hline
\end{tabular}


El segundo factor, sociocultural, quedó integrado por 12 variables, todas ellas referidas a indicadores culturales (ver tabla V). Su varianza explicada es de 10.12 por 100 .

\section{TABLA V}

Factor 2: Nivel sociocultural

\begin{tabular}{ll}
\hline \multicolumn{1}{c}{ Variables } & $\begin{array}{c}\mathrm{C} . \\
\text { factoriales }\end{array}$ \\
\hline Lectura de periódicos del padre & .614 \\
Idioma que el padre habla con el hijo/a & .610 \\
Lectura de periódicos de la madre & .576 \\
Asistencia al cine del padre & .565 \\
Idioma que la madre habla con el hijo/a & .562 \\
Programas de TV que ve el padre & .561 \\
N.9 de secciones del periódico que lee el padre & .536 \\
N.9 de secciones del periódico que lee la madre & .519 \\
Lectura de libros del padre & .513 \\
Programas de TV que ve la madre & .443 \\
Juguetes educativos de los hijos/as & .390 \\
Asistencias al cine de la madre & .375 \\
\hline
\end{tabular}

En función de los resultados de este análisis describimos los elementos típicos que caracterizan a las distintas clases sociales, y realizando un análisis comparativo entre estos grupos comprobamos que donde se encontraban las mayores diferencias era en aquellas variables que se referían al nivel socioeconómico, lo cual confirma el hecho de que el factor básico para diferenciar entre grupos sociales es el económico. También observamos que el nivel sociocultural es un factor diferenciador importante, aunque en nuestra muestra las mayores diferencias con respecto a este factor se dan entre la clase social baja y alta.

\section{B. Prueba «t» de significatividad de diferencias entre grupos \\ - Clase Social Baja y Alta}

A nivel global observamos que las diferencias entre estos dos grupos son significativas en todos los subtests de la Batería I.T.P.A. a un n.s. que oscila entre 0.000 y 0.006 , excepto en el proceso de recepción auditiva y visual (ver tabla VI).

TABLA VI

\begin{tabular}{lc}
\hline \multicolumn{2}{c}{ Clase social baja-Clase social alta } \\
\hline \multicolumn{1}{c}{ Subrest del ITPA } & n.s. \\
\hline Asociación visual & 0.000 \\
Asociación auditiva & 0.000 \\
Expresión verbal & 0.002 \\
Expresión motora & 0.006 \\
Integración gramatical & 0.000 \\
Integración visual & 0.002 \\
Memoria S. auditiva & 0.003 \\
Memoria S. visual & 0.002 \\
\hline
\end{tabular}


Quiere esto decir que en la mayoría de las funciones lingüisticas, cognitivas, peceptivas y de memoria, evaluadas a través del I.T.P.A., los sujetos de estos grupos sociales presentan diferencias significativas y, en todos los casos, éstas están a favor del grupo de clase social alta. Específicamente se observa que los niños/as pertenecientes a familias socioculturalmente favorecidas obtienen mejores resultados en aquellas habilidades $y / o$ capacidades necesarias para relacionar conceptos, percepciones y símbolos, expresar ideas verbal o gestualmente, completar las partes que faltan en un todo - dibujo y/o frase- y recordar de forma inmediata material no significativo.

\section{- Clase Social Baja y Media}

Las diferencias significativas que encontramos entre estos grupos se refieren a los subtests de comprensión visual, asociación auditiva, integración gramatical y expresión motora (ver tabla VII).

TABLA VII

\begin{tabular}{|c|c|}
\hline \multicolumn{2}{|c|}{ Clase social baja-Clase social media } \\
\hline Subtest del ITPA & n.s. \\
\hline Comprensión visual & 0.051 \\
\hline Asociación auditiva & 0.003 \\
\hline Expresión motora & 0.030 \\
\hline Integración gramatical & 0.013 \\
\hline
\end{tabular}

Estos sujetos se diferencian en la habilidad para obtener significado de símbolos visuales, en la capacidad para relacionar conceptos y símbolos cuando el material se presenta oralmente, en la capacidad para utilizar la sintaxis en una tarea de completar frases mediante dibujos y en la habilidad para expresar ideas gestualmente.

\section{- Clase Social Media y Alta}

El grupo de sujetos que pertenece a la clase social media de nuestra muestra se diferencia de la clase alta fundamentalmente en base a indicadores socioeconómicos. Las medidas del nivel sociocultural son bastante semejantes en los dos grupos; este hecho creemos que puede estar explicando que únicamente obtengamos diferencias significativas en los subtests de memoria secuencial auditiva, asociación visual e integración gramatical (ver tabla VIII).

TABLA VIII

\begin{tabular}{ll}
\hline \multicolumn{2}{c}{ Clase social alta-Clase social media } \\
\hline \multicolumn{1}{c}{ Subtest del ITPA } & n.s. \\
\hline Asociación visual & 0.035 \\
Integración gramatical & 0.026 \\
Memoria S. auditiva & 0.042 \\
\hline
\end{tabular}


Las diferencias observadas se refieren a la capacidad para relacionar conceptos presentados visualmente, a la habilidad para usar la gramática de modo automático y para el recuerdo inmediato de material no significativo a través de la repetición de series.

Las diferencias encontradas en los subtests del I.T.P.A., comentadas hasta aquí, pueden estar indicando que los niveles socioeconómicos y socioculturales, definitorios de las distintas clases sociales, inciden de manera indirecta o directa sobre algunas de las formas lingüísticas analizadas, lo cual conlleva a que el rendimiento de los niños de los grupos sociales más favorecidos en las funciones lingüísticas sea superior al de los sujetos desfavorecidos socioculturalmente dado el entorno social menos estimulante en que se desarrolla.

\section{Análisis de Regresión Múltiple}

El último paso que realizamos dentro de los análisis estadísticos inferenciales fue el Análisis de Regresión Múltiple, con el objetivo de que nos posibilitase conocer cuáles de las variables que, en un principio, fueron relevantes en el Análisis Factorial, están ahora afectando, determinando o prediciendo un determinado rendimiento en la ejecución lingüistica de los sujetos de la muestra.

El fin último que pretendemos lograr con este análisis es evaluar la dependencia de una variable dependiente - compuesta por los subtests de la Bateria I.T.P.A. - de unas variables independientes - integradas por los niveles socioeconómico y cultural.

Los resultados alcanzados mediante este Análisis de Regresión nos permiten observar que las variables independientes que predicen un determina-

\section{TABLA IX}

Variables independientes que influyen en los resultados de la ejecución lingüistica en los subtests del I. T. P. A.

\begin{tabular}{lll}
\hline \multicolumn{1}{c}{ Subtest } & \multicolumn{1}{c}{ V.l. } & n.s. \\
\hline Comprensión visual & Número secciones periódico padre & 0.040 \\
Asociación auditiva & Número secciones periódico padre & 0.016 \\
& Número programas TV padre & 0.020 \\
& Frecuencia asistencia cine madre & 0.027 \\
& Actividades culturales madre & 0.005 \\
& Idioma que el padre habla con hijo/a & 0.032 \\
Asociación visual & Frecuencia asistencia cine madre & 0.034 \\
& Actividades culturales madre & 0.033 \\
Expresión verbal & Idioma que el padre habla con hijo/a & 0.016 \\
Integración gramatical & Estudios de la madre & 0.034 \\
& Frecuencia lectura periódico padre & 0.017 \\
& Frecuencia asistencia cine madre & 0.003 \\
& Actividades culturales padre & 0.018 \\
& Actividades culturales madre & 0.002 \\
Integración visual & Idioma que el padre habla con hijo/a & 0.010 \\
& Presupuesto educativo & 0.048 \\
Memoria S. auditiva & Número habitaciones vivienda & 0.048 \\
& Número secciones periódico padre & 0.005 \\
& Número secciones periódico madre & 0.030 \\
& Actividades culturales padre & 0.032 \\
& Actividades culturales madre & 0.018 \\
\hline
\end{tabular}


do rendimiento en el lenguaje son mayoritariamente variables socioculturales, concretamente las siguientes: número de secciones del periódico que leen los padres, número de programas de TV que ve el padre, frecuencia en la asistencia al cine de la madre, frecuencia en la asistencia a actividades culturales de los padres e idioma que el padre habla con sus hijos. Por lo que respecta al factor socioeconómico sólo las variables estudios de la madre, número de habitaciones de la vivienda y presupuesto educativo, determinan y/o predicen las puntuaciones de los sujetos en los subtests de expresión verbal e integración visual (ver tabla IX).

Teniendo presentes los estadísticos descriptivos de cada una de las variables citadas, comprobamos que éstas predicen o determinan los distintos subtests de la Batería I.T.P.A. cuando toman valores que se corresponden a los que definen los grupos sociales medio y alto, lo cual corrobora los resultados obtenidos en la Prueba «t» de Student.

\section{CONCLUSIONES}

Las conclusiones extraídas de esta investigación pueden formularse en los siguientes términos:

1. De modo general, coincidimos con otros autores (B. Bernstein, 1961, 1975; D. Lawton, 1963; F. Williams, y B. Woods, 1970; M. Golden, y B. Birns, 1976) al afirmar que existen diferencias lingüísticas significativas según la procedencia social de los sujetos. Estas diferencias pueden concretizarse del modo siguiente:

a) Comparando la clase social baja y alta obtenemos diferencias significativas en todos los subtests de la batería I.T.P.A., excepto en el proceso de recepción.

b) Los sujetos de clase social media obtienen mejores resultados que sus iguales de clase baja en aquellas tareas que requieren de las habilidades para relacionar conceptos, comprender un significado presentado oralmente, usar la gramática de manera automática y expresar significados mediante gestos motores.

c) Las diferencias lingüísticas significativas entre los niños de clase media y alta se refieren a la habilidad para relacionar conceptos presentados visualmente, usar la gramática y para el recuerdo inmediato de material no significativo presentado oralmente.

Esto nos permite afirmar la H.1 y parcialmente las subhipótesis $\mathrm{H} .1 \mathrm{a}, \mathrm{b}$ y c, puesto que las diferencias en la ejecución lingüística son más acusadas en la medida en que el nivel socioeconómico y cultural de los sujetos de la muestra presenta mayor variabilidad.

2. Por último, afirmamos que de entre los indicadores sociales objetivos de clase social, los socioculturales son los que afectan en mayor medida al rendimiento en lenguaje, lo cual nos permite confirmar la H.2. Estas variables se refieren a las actividades culturales de los padres, a la posesión de información, es decir, a las posibilidades de adquirir y disfrutar de oportunidades para conseguir un determinado bagaje sociocultural.

Para explicar esto, nos apoyamos en las tesis de Basil Bernstein que afirma que el lenguaje refleja los esquemas de interacción y de acción que están en la base de los diferentes grupos sociales. Queremos decir que el ambiente que rodea al niño, específicamente el entorno sociocultural y lingüístico, favorece, en el mejor de los casos, aquellas capacidades y habilidades que se requieren para lograr una ejecución lingüística óptima y, correlativamente, éxito escolar. 
La solución al problema de las desigualdades lingüísticas de los diferentes miembros de las clases sociales puede venir de una política social que contribuya a paliar las dificultades de las familias menos favorecidas socioculturalmente, abordando el contexto ambiental y lingüístico en que vive el niño, y posibilitando un medio cultural más enriquecedor. Por su parte, también la escuela puede y debe desempeñar un papel importante. El rol de la escuela es básico porque en sus manos está el poder remediar, en parte, las condiciones lingüísticas en que están inmersos los niños menos favorecidos, para ello debe evitar la ruptura que se produce entre el mundo simbólico familiar de estos niños y el escolar. Debe luchar contra las desigualdades socioculturales insertando el contexto ambiental en el que se desarrolla el alumno en el marco escolar, y convertirse en la escuela de cada uno transmitiendo una educación personalizada, diferenciada e integradora, en aras de una educación más justa.

\section{Resumen}

En este articulo se realiza una aproximación al estudio y evaluación del lenguaje desde una perspectiva psicosocial. El objetivo prioritario de esta investigación es conocer y exponer la importancia y relevancia que poseen ciertas variables socioeconómicas culturales, tipificadas como indicadores sociales, sobre el rendimiento en lenguaje, evaluado a través del I.T.P.A., de una muestra de escolares del Ciclo Medio de la E.G.B. en Galicia.

\section{Summary}

We deal with an approximation to study and measure of language from a psychosocial perpsypective. The main aim of these research is to find out and sbow the importance that some social, economical and cultural variables bave as an social markers, about the performance on the language, evaluated through of the I.T.P.A., in a sample of students of E.G.B. in Galicia.

\section{Résumé}

Nous avons réalisé une approximation à l'étude et à l'évaluation du langage selon une perspective psychosociale. L'objectif principal de cette recherche est de connaître et d'expliquer l'importance et la rélevance qui ont quelques variables socioéconomiques et culturelles, définées com. me indicateurs sociaux et qui conditionnent le rendement du langage, étudié d'accord avec le I.T.P.A., dans une échantillon dielèves de l'E.G.B. en Galicia.

\section{Referencias}

ARAM, D.: Code in context: the descriptive speech of seven year old children. Londres: Institute of Education, 1975.

BERNSTEIN, B: «Language and social class». British J. Sociol., 1960, 2, 271-276.

Bernstein, B.: «Social class and linguistic development. A theory of social learning», en A. H. Harley, J. Floud, y C. A. Anderson, (eds.):Education, economy and society. Nueva York: Free Press, 1961.

BERNSTEIN, B.: Class, codes and control (vol. 2): Applied studies towards a sociology of language. Londres: Routtledge and Kegan, 1972.

Bernstein, B.: Langage et classes sociales. París: Minuit, 1975.

BRONCKART, J. P.: «Adquisición del lenguaje y desarrollo cognitivo», en Varios: La génesis del lenguaje. Madrid: Pablo del Río, 1977.

Giles, H.; Robinson, W. P., y Smith, P. H. M.: Language. Social Psychology Perspectives. Oxford: Pergamon Press, 1980.

GolDEN, M., y BIRNS, B.: «Social class and infant intelligence», en M. Lewis:Origin of intelligence. Nueva York: Wiley, 1976.

HallidAY, M. A. K.: Language as social semiotic. The social interpretation of language and meaning. Londres: Arnold, 1978.

LAwTON, D:: «Social class, differences in language development: A sutdy of some sample of written work». Language and Speech, 1973, 6, 120-132. 
LEONTIEv, A. N.: «Social and natural in semiotics», en Morton, J. (ed.): Biological and social factors in psycholinguistics. Londres: Logo, 1971.

MaYor, J.: Psicología del Lenguaje y del Pensamiento. Madrid: UNED, 1984.

SALZINGER, K.: «Ecolinguistics: a radical behavior theory approach to language behavior», en B. R. Aaronson, y R. W. Rieber, (eds.): Psycholinguistic research. Hillsdale: LEA, 1979.

SORIANo LloReNT, A.: «El entorno socioeconómico como variable controlada en las pruebas sobre lenguaje y personalidad en niños de siete a nueve años». Anuario de Psicología, 1972, 6, 62-115.

TURNER, G., y PICKWANCE, R.: «Social Class differences in the expression of uncertainty in five-year old children's speechs. Language and Speech, 1971, 14, 3, 3-25.

TURNER, G.: «Social class and children's language of control at ages five and seven», en B. Bernstein: Class, codes and control. Vol. 2. Londres: Routledge and Kegan, 1972.

WERTSCK, J. V.: Recent trends in Soviet psycholinguistics. Nueva York: Thorpe, 1978.

WILIAMS, F., y NAREMORE, R.: «On the functional analysis of social class differences in modes of speech». Speech Monograph, 1969, 36, 77-102.

WILLIAMS, F., y WOODS, B.: «Negro children's speech some social class differences in word predictability». Language and Speech, 1970, 13, 141-150. 